\title{
An experimental framework to capture the flow dynamics of droplets expelled by a sneeze
}

\author{
Prateek Bahl ${ }^{1}\left[\right.$ Charitha M. de Silva ${ }^{1}(1) \cdot$ Abrar Ahmad Chughtai $^{2} \cdot$ C. Raina Maclntyre ${ }^{3} \cdot$ Con Doolan $^{1}$
}

Received: 2 February 2020 / Revised: 1 July 2020 / Accepted: 4 July 2020 / Published online: 18 July 2020

(c) Springer-Verlag GmbH Germany, part of Springer Nature 2020

\begin{abstract}
Respiratory activities such as sneezing generate pathogen laden droplets that can deposit in the respiratory tract of a susceptible host to initiate infection. The extent of spread of these droplets determines the safe distance between a patient and health care worker. Here, we have presented a method to visualize the droplets expelled by a sneeze using light-sheet illumination. This method of visualization provides images that clearly resolve the velocities of droplets with minimal overlapping trajectories, towards understanding their flow dynamics. Furthermore, we present the image processing techniques required to perform accurate Particle Tracking Velocimetry to understand the motion of expelled droplets. Flow fields are presented from applying this methodology over multiple sneezes which reveal that less than $1 \%$ of droplets expelled travel at velocities greater than $10 \mathrm{~m} / \mathrm{s}$ and almost $80 \%$ of droplets travel at velocities less than $5 \mathrm{~m} / \mathrm{s}$. Furthermore, we observe that some droplets are generated by ligament breakup outside the mouth and some are generated within the respiratory tract.
\end{abstract}

\section{Graphic abstract}
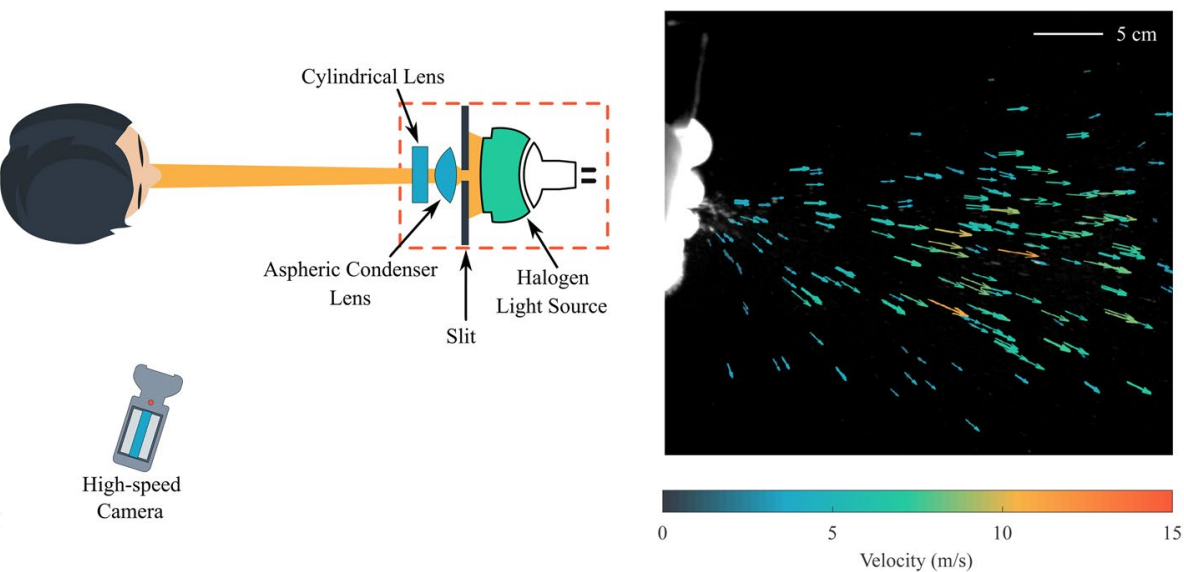

Prateek Bahl

prateek.bahl@protonmail.com

1 School of Mechanical and Manufacturing Engineering, UNSW Sydney, Kensington, NSW 2052, Australia

2 School of Public Health and Community Medicine, UNSW Sydney, Kensington, NSW 2052, Australia

3 Biosecurity Program, The Kirby Institute, UNSW Sydney, Kensington, NSW 2052, Australia

\section{Introduction}

Coughing and sneezing are often considered as aerosol generating actions. Sneezing is characterized by a sudden involuntary expulsion of respiratory and salivary fluid along with air, from the mouth and nose, due to irritation of the mucus membrane. Sneezes expel pathogen-laden droplets of respiratory and salivary fluid that can deposit in the respiratory tract of susceptible hosts in the near-vicinity, and that may initiate the infection. Although both coughing and sneezing are sudden expulsions of air and respiratory tract fluid, 
studies have shown that they have certain characteristics. Specifically, initial studies by Duguid (1944) and Jennison (1942) showed that peak velocity of droplets expelled during a sneeze is significantly higher than that generated during cough and the number of droplets expelled during a sneeze are also much higher than those produced during coughing. A more recent study by Scharfman et al. (2016) showed that even the mechanism of droplet formation differs between a cough and a sneeze. As a consequence of these properties, sneezing could be a major contributor to 'droplet' transmission as defined by World Health Organization (2014), which is believed to occur when a person is within $1 \mathrm{~m}$ from someone who has respiratory symptoms (e.g., coughing or sneezing). However, most prior research on infection transmission has been focused on coughing with sneezing receiving much less attention comparatively (Zhu et al. 2006; Gupta et al. 2009; Vansciver et al. 2011; Lee et al. 2019). A reason for this bias could be the frequency of coughing, which could be up to 15 times higher in certain diseases (Loudon and Brown 1967; Dick et al. 1987). Moreover, it is also harder to induce a controlled sneeze as compared to cough for experimental studies.

The infection control guidelines for the prevention of respiratory transmission makes an assumption on the safe distance between patient and health care worker based on the extent of spread of droplets expelled during respiratory events such as coughing and sneezing, and 1-2 m distance is considered safe to prevent from 'droplet' transmission. A recent review by Bahl et al. (2020) found that the evidence for this safe distance is very sparse. Moreover, for coronavirus disease 2019 (COVID-19) pandemic the spatial separation limit for donning Personal Protective Equipment (PPE), as defined by WHO is just $1 \mathrm{~m}$ (World Health Organization 2020). Therefore, understanding the dynamics of respiratory events is key to guiding infection control policy and practice in the healthcare setting, including the use of PPE to provide a barrier against large droplets and aerosols (MacIntyre et al. 2014). Modelling studies by Xie et al. (2007) and Parienta et al. (2011) have shown that the properties of the expiratory fluid and the velocity of expiration, plays a major role in determining the extent of droplet spread. Hence, a precise measurement of the velocity of expelled droplets is essential to better understand the transmission of infection.

A few studies have tried to study the flow dynamics of sneezes using various techniques. Nishimura et al. (2013) used volumetric illumination and a high-speed imaging system to capture sneeze flow at $300 \mathrm{fps}$. The study employed Particle Image Velocimetry to analyse the flow and found the initial velocity to be greater than $6 \mathrm{~m} / \mathrm{s}$. It was also concluded that the reach of a sneeze is almost three times that of cough however, this study was limited to a single subject. Tang et al. (2013) collected data from 6 subjects using shadow-graph imaging at a frame rate of $2000 \mathrm{fps}$ and reported a maximum velocity of $4.5 \mathrm{~m} / \mathrm{s}$. Bourouiba et al. (2014) provided a theoretical model, which was developed using high-speed sneeze visualization, analogous water tank experiments and a particle-laden cloud model, to predict the extent of pathogen spread. It was concluded that the turbulent cloud expelled during a sneeze can extend the reach of pathogen laden droplets. Scharfman et al. (2016) used high-speed cameras to capture close-range droplets using diffused white backlighting and concluded that droplets predominantly form at the exit of the mouth by a sequence of fragmentation processes. It was also concluded that the viscoelasticity of expiratory fluid is a major factor in determining the size distribution of large droplets. The maximum velocity of droplets reported in this study was $14 \mathrm{~m} / \mathrm{s}$ and a velocity of up to $35 \mathrm{~m} / \mathrm{s}$ was reported for ligaments, which subsequently breaks to form large droplets. A recent visualization of sneeze cloud by Bourouiba (2020) showed that

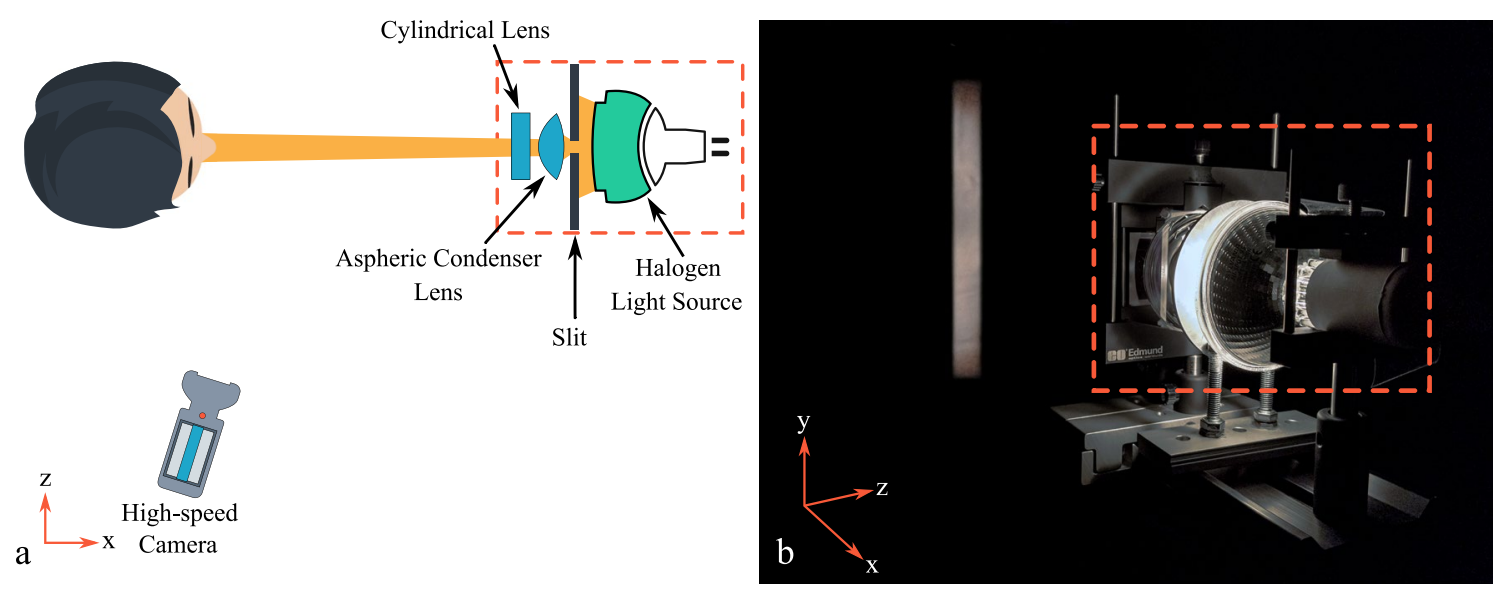

Fig. 1 Experimental setup used to capture droplets expelled during a sneeze. a Schematic showing top view of the setup. $\mathbf{b}$ A picture of the experimental setup showing the vertical light sheet ( $x y$ plane) used in the present study 
turbulent gas cloud with pathogen laden droplets can travel up to $8 \mathrm{~m}$ away from the subject.

Since droplet precaution guidelines for infection control rely on the extent of droplet spread (Siegel et al. 2007), and appears to assume no difference between droplets expelled by a cough and sneeze, it is important to understand the dynamics of individual droplets. We note that most of the published literature on sneeze flow dynamics uses volume illumination for flow visualization, coupled with a single camera. However, with volume illumination, there is an overlap of droplet trajectories, and the movement of particles along the camera axis can lead to difficulty in precisely detecting the velocity of individual particles. Other works include measurements by Scharfman (2016) which used a multi-camera arrangement together with back illumination.

For single camera measurements, light sheet illumination allows to precisely locate particles in a slice of the flow-field which are not masked by the presence of particles along the camera axis. Studies by Zhu et al. (2006) and Vansciver et al. (2011) used laser sheets for Particle Image Velocimetry (PIV) to understand cough flow by making a chamber with PIV setup in which volunteers cough through an opening. Since in the case of sneezing, head movement is involved, and it is much more difficult to control a sneeze, this kind of approach is not possible to visualize sneeze flow.

Accordingly, in this study, we present a set of experiments employing light sheet illumination to capture individual droplets with minimal overlapping along the axis of the camera. The images captured are then processed using Particle Tracking Velocimetry techniques to obtain precise individual droplet motion. In Sect. 2, we describe the experimental methods to capture the sneeze flow using light sheet illumination and a high-speed camera. The image processing required for the study is explained in Sect. 3. Sections 4 and 5 present the analysis of the results.

We note, to complete the study approval was obtained from the University of New South Wales Human Research Ethics Committee [HC180830]. All the participants were healthy and non-smokers and provided written consent before participating in the study.

\section{Experiments}

Droplets expelled during a sneeze were captured by employing a high-speed camera and light sheet illumination. The use of light sheet illumination and high-speed motion capture was important to resolve the details of individual droplets expelled during a sneeze. To this end, a vertical ( $x y)$ plane was illuminated using a halogen light source (see Sect. 2.1 for further details). The head of the subject was positioned in front of a black backdrop and it was aligned in such a way that subject was facing the light source and the light plane was illuminating the middle of the face (Fig. 1). Once the head was positioned the subject used a tissue paper to simulate the mucus membrane of the nasal cavity to induce a sneeze, which was captured using a high-speed camera.

\subsection{Light sheet illumination}

For the present study, a light sheet is employed to illuminate the flow of a sneeze in a vertical ( $x y$ ) slice. Since a single camera is used for 2D measurements, illuminating a slice of flow eliminates the averaging of the particle motion in the direction perpendicular to the light sheet when compared to volume illumination. This results in clearly resolved particle trajectories that are suitable for particle tracking. We note due to safety concerns the use of a laser sheet to visualize sneeze flow is not possible, hence, the use of an alternate light source to make a light sheet is essential for this study.

To this end, a halogen spotlight with a nominal beam divergence angle of $10^{\circ}$ was used. To form a light sheet, a $5 \mathrm{~mm}$ wide and $80 \mathrm{~mm}$ long slit made out of aluminium sheet was fixed in front of the light source and an aspheric condenser lens with a diameter of $80 \mathrm{~mm}$ and a focal length of $59 \mathrm{~mm}$ was positioned at a distance of $59 \mathrm{~mm}$ from the slit to collimate the diverging light beam. Thereafter, a $50 \times 50 \mathrm{~mm}$ plano-concave cylindrical lens with a focal length of $38 \mathrm{~mm}$ was used to diverge the beam in the vertical direction. This configuration provided a horizontal divergence angle of $1.4^{\circ}$ (see Fig. 1), which was sufficient to capture the droplets and minimize the haze due to expelled aerosols. With this arrangement, we achieved a sheet thickness of $25 \pm 2 \mathrm{~mm}$ in the field of view captured.

\subsection{High-speed motion capture}

The duration of a sneeze is approximately $150-200 \mathrm{~ms}$ (Bourouiba et al. 2014; Scharfman et al. 2016) hence it is essential to use high-speed video to resolve the droplet motion. Accordingly, a high-speed monochrome camera (nac MEMRECAM HX-7s) was used to record high-speed videos of sneezes at a resolution of $1920 \times 1080$ pixels and a frame rate of 1500 frames-per-sec (fps). However, at the desired exposure time at $1500 \mathrm{fps}$, the intensity level from a single light source was deemed insufficient to resolve the droplets if the camera is perpendicular to the light sheet due to the mie-scattering properties of the particles (Tropea et al. 2007). To overcome this, the camera was positioned at an angle from the direction of the light sheet. We note, recording videos at an angle introduces perspective distortion, hence, the camera was positioned at an angle of $80^{\circ}$ as a balance between adequate light scattering and the level of perspective distortion. Another drawback of using the camera at an angle is the inclination of the focal plane with 

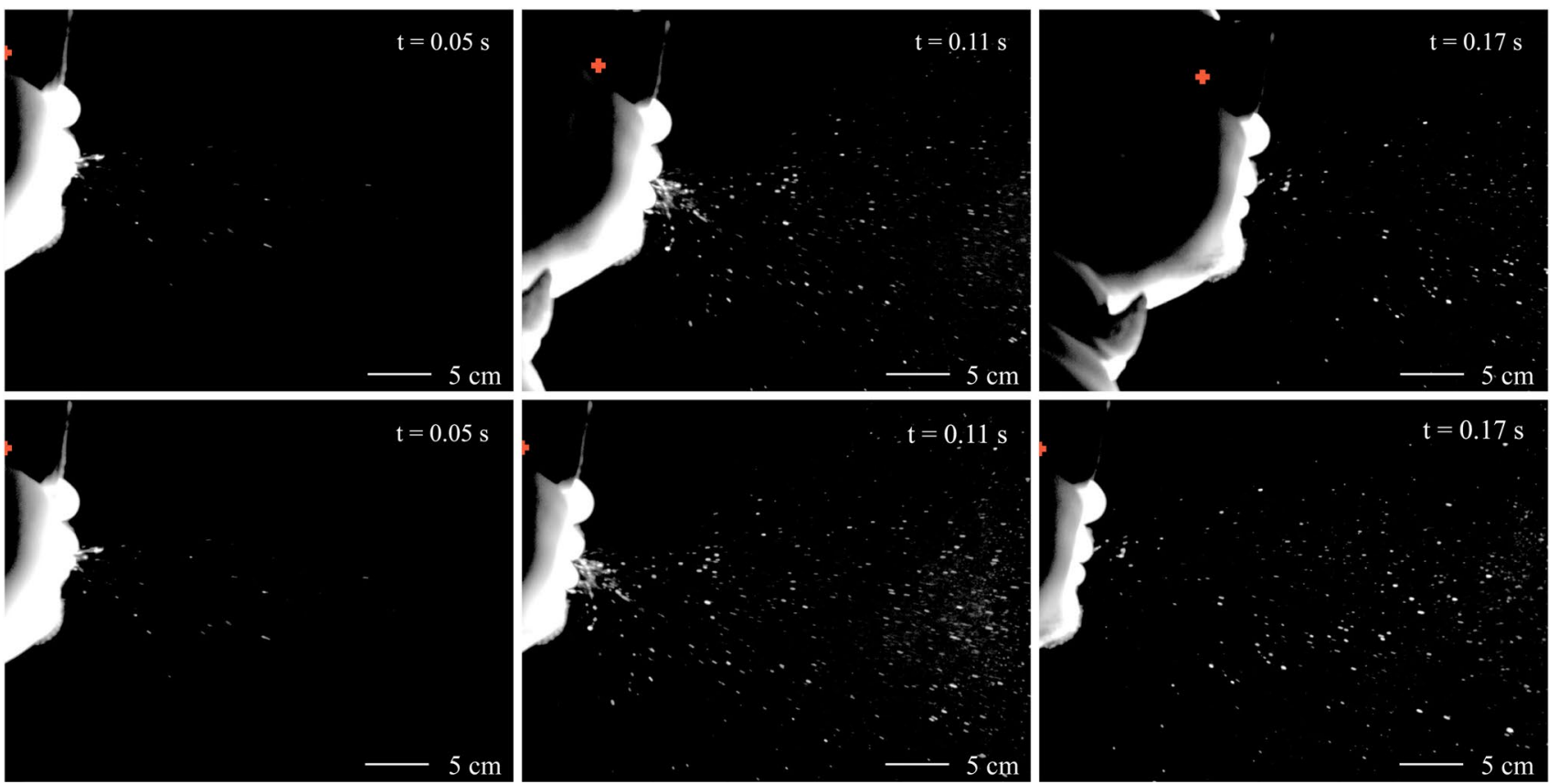

Fig. 2 Stabilizing movement of head movement. Top row shows the sneeze developing in time and the red marker shows the mid-point of the tracked feature. Bottom row shows the sneeze development after fixing the mid-point of the tracked feature

respect to the image plane, which resulted in droplets going out of focus. Hence, we employed an f-stop of f4 to ensure all particulates remain in focus.

\section{Image processing and analysis}

To perform particle tracking, the image sequences were first passed through a sequence of pre-processing steps. In the present study, this was performed in multiple steps. First, we removed the background noise through a background subtraction from all the images in the sequence. Next, the perspective distortion that was introduced through the angled view of the camera was corrected (detailed in Sect. 3.1). Finally, to isolate the motion of the expelled droplets, the motion of the subject's head was removed by applying a 2-axis stabilization (detailed in Sect. 3.2). We note, after this process some minimal movement in the face was still present due to muscle-flexing. This was eliminated by applying a dynamic mask to all the images as part of post-processing of the data sets (detailed in Sect. 3.4).

\subsection{Removing perspective distortion}

To obtain a precise conversion from pixel coordinates to real coordinates, a calibration target with $27 \times 29$ dots and a dot spacing of $5 \mathrm{~mm}$ was employed. Furthermore, this removed any perspective distortion in the images. Specifically, prior to capturing data, an image of the calibration target, placed in the focal range of the camera and aligned with the light sheet was taken. A transform was then created for this calibration image to remove the perspective distortion. This transformation was then applied to all the images in the sequence (De Silva et al. 2012).

\subsection{Stabilizing head movement}

Stabilizing the movement of the head is essential to isolate the motion to the expelled droplets in the image sequence and to determine the absolute velocity of expelled droplets alone. For stabilizing head movement, a feature in all the images of the image sequence was tracked (see Fig. 2). This was done using a template matching algorithm that utilised 2D normalized cross-correlation (Lewis 1995). This procedure was chosen as it is less sensitive to variations in lighting.

Once the feature tracking was completed successfully the midpoint of the tracked feature was forced to be fixed at a particular location across all the images. This was done by dynamically shifting the whole image array based on the vertical and horizontal displacement of the tracked feature. Thereafter, images were cropped based on the field of view available in the image at the final location of the head.

The output of this process is shown in Fig. 2. The first image sequence (top row) shows the midpoint of the tracked feature using normalized cross-correlation (marked with a red marker). And the second image (bottom row) sequence 


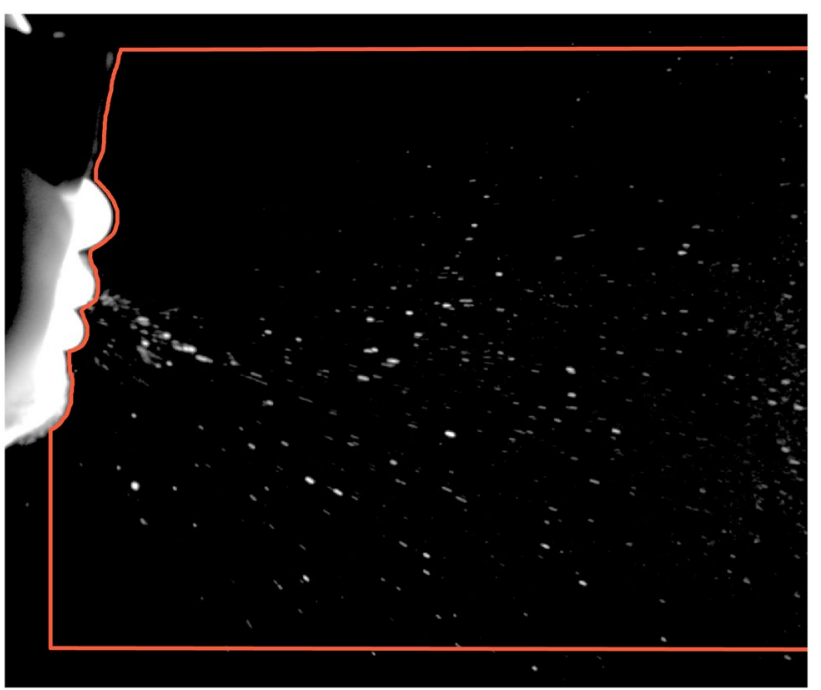

Fig. 3 Example of facial contour detected to generate a mask for removing erroneous velocity vectors. Red line shows the mask applied on a single frame of the image sequence

shows the tracked feature at a fixed position to stabilize the movement of the head.

\subsection{Particle tracking velocimetry}

After pre-processing the images, Particle Tracking Velocimetry (PTV) was performed on the image sequence to understand the flow dynamics of expelled droplets. Lavision® Davis 8.4 was used to process the data. Specifically, due to the high seeding density in certain regions of the image, the procedure first performs a Particle Image Velocity (PIV) pass on the image sequence to obtain a velocity field estimate and then tracks individual particles using PTV algorithm (Cowen and Monismith 1997). The interrogation window used for PIV step starts with an initial interrogation window size of $96 \times 96$ pixels and final size of $48 \times 48$ pixels with an overlap of $75 \%$. For the PTV step, a particle size range between 6 pixels to 40 pixels was employed. This larger range was chosen to cover the range of droplet sizes present in the image sequence of the sneeze flow. The PTV algorithm tracks each particle independently and the interrogation window size is only used for an initial displacement estimate through PIV, which is then refined in the particle tracking algorithm. The hybrid approach used for particle tracking provides high accuracy with a maximum estimation error of \pm 0.5 pixels (Cowen and Monismith 1997).

\subsection{Dynamic masking}

A dynamic mask was employed in order to remove erroneous velocity data in the near-vicinity of the subject's face. To this end, a facial contour of the subject was detected and any velocity data outside the area of interest was discarded (Fig. 3).

To find the facial contour, every image in the sequence was first pre-processed using pixel intensity manipulation to accentuate the edge of the face. Thereafter, a 2D convolution was performed on the image using $\left[\begin{array}{ll}-2 & 2\end{array}\right]$ and $\left[\begin{array}{c}-2 \\ 2\end{array}\right]$ gradient filters to obtain horizontal and vertical gradients. Once the gradients were calculated a Fuzzy Interface System (FIS) was created for edge detection with horizontal and vertical image gradients as input (MATLAB 2019). Specifically, one Gaussian membership function was defined for each input and two triangular membership functions were defined for the output. The FIS system makes the pixel white in the output image if the image gradient in both horizontal and vertical directions was zero. If there was any non-zero gradient, horizontal or vertical, at the pixel location then it meant that pixel is on the edge and the output was black. This process was performed for each row of pixels in the image to find the facial contour. Once facial contour was determined, each pixel row was analysed to get a unique edge location. This spatial information of edge location in each image was
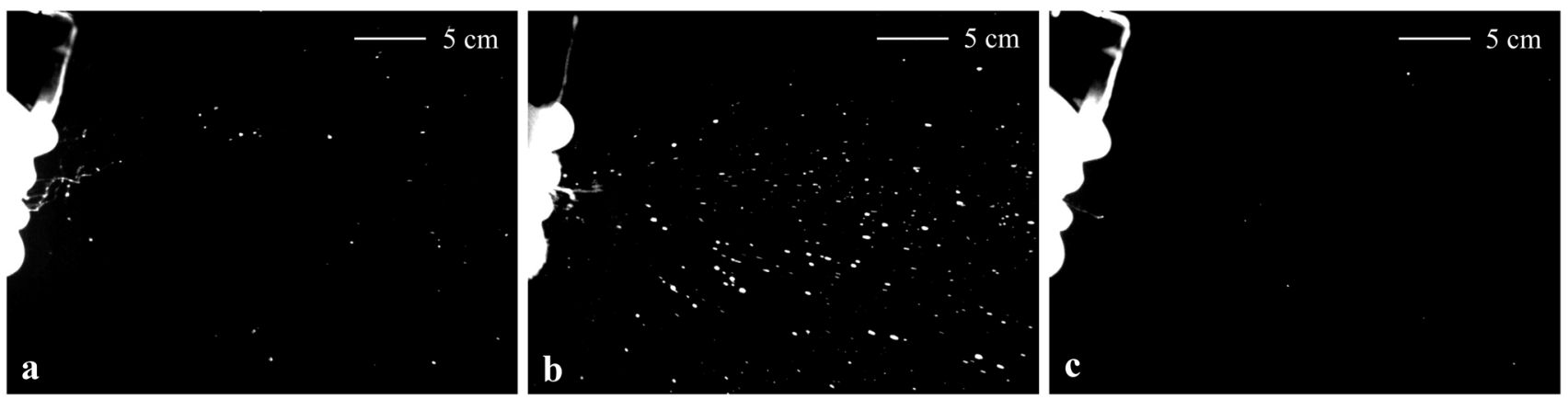

Fig. 4 Variation in expelled droplets of 3 different sneezes. Snapshots shown are at $150 \mathrm{~ms}$ after the onset of sneeze. Here, a and $\mathbf{c}$ are snapshots of different sneeze sequence from same individual 

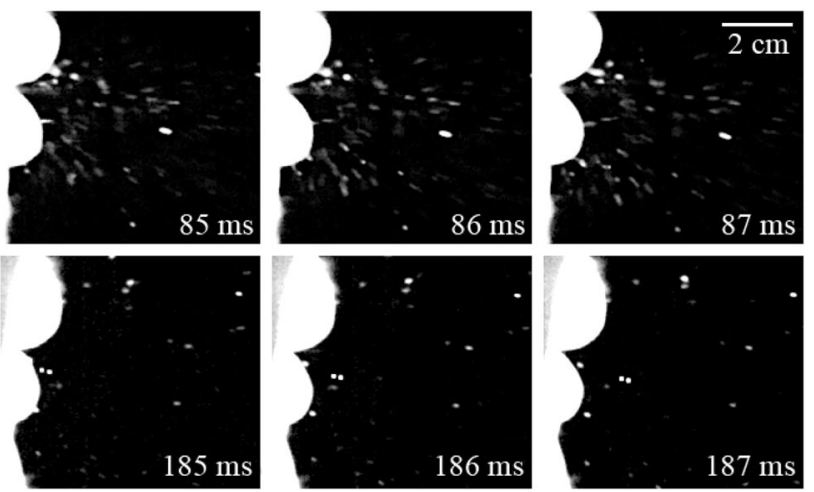

Fig. 5 Small droplets expelled from the mouth at $85-87 \mathrm{~ms}$ and at 185-187 ms for sneeze 2

then combined in a single file which acts as a dynamic mask for that particular image sequence.

\section{Examination of sneeze flow-fields}

To characterise the flow-field of the sneeze, the aforementioned methodology was applied to a set of sneezes captured. Although the sample size was limited to 2 subjects, significant differences were observed between sneezes, even from the same subject, in terms of droplet velocity and duration of the sneeze. Furthermore, the number of expelled droplets observed also varied significantly, which highlights the importance of examining a large set of sneezes towards fully characterising the behaviour of sneeze flow.

To visualize these differences, 3 independent sneezes are presented in Fig. 4. Here, sneeze 1, in Fig. 4a and sneeze 3 , in Fig. 4c are from the same subject yet still exhibit differences in terms of droplets expelled and the duration of a sneeze. Specifically, more droplets can be observed in sneeze 1 when compared to sneeze 3 . Another phenomenon we observed in sneeze 1 is the presence of ligaments close to mouth which appears to breaks into individual droplets with time. This could be due to various hydrodynamic instabilities, as highlighted by Scharfman et al. (2016), where they observed similar phenomena through flow visualisation experiments. Sneeze 2, in Fig. $4 \mathrm{~b}$ is from a different subject and it can be observed that there is presence of ligaments near the mouth exit, however, most detected small droplets appear to be expelled directly out of the mouth (Fig. 5). Which implies that although large droplets are formed at the exit of the mouth by ligament breakup, small droplets are formed within the respiratory tract. Another important observation is that although ligaments are present in both sneeze 1 and sneeze 2 in the latter we observe a rapid conversion of ligaments to droplets. This could be due to variation in airflow and viscoelasticity of salivary fluid of different sneezes (Fig. 4).

Figure 6 shows the distribution of velocity vectors for all three sneezes and it can be observed that sneeze 1 and sneeze 2 exhibit more vectors in the range of $5-10 \mathrm{~m} / \mathrm{s}$ as compared to sneeze 3 . In sneeze 3 , most of the vectors are in the range of $0-5 \mathrm{~m} / \mathrm{s}$. These differences in velocity vector distributions, specifically in sneeze 3 could be due to the variation in the droplets captured in the slice of flow, as the droplets visible in sneeze 3 are much less compared to other 2 sneezes, which can be observed in Fig. 4. We note that ligaments are treated as single large particles in the particle tracking approach used and the velocity vectors associated with ligaments are also included in the distribution shown in Fig. 6.

The set of images in Fig. 7 shows the development of sneeze 2 in time. The onset of the sneeze is determined by visually detecting the start of airflow from the mouth or nose of the subject. In this case, the sneeze lasted for approximately $190 \mathrm{~ms}$ and the maximum observed velocity of particles expelled is approximately $14 \mathrm{~m} / \mathrm{s}$.

We note that although the methodology employed resolved droplet dynamics only over a vertical $(x y)$ slice
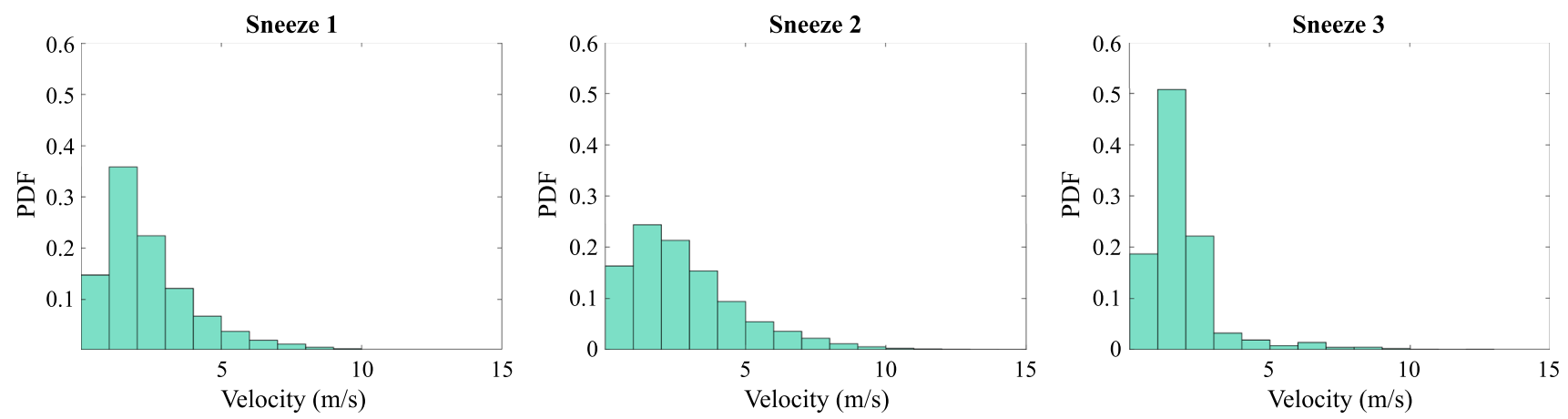

Fig. 6 Probability distribution of velocity vectors obtained by particle tracking for the complete duration and field of view of sneeze 1 , sneeze 2 and sneeze 3 

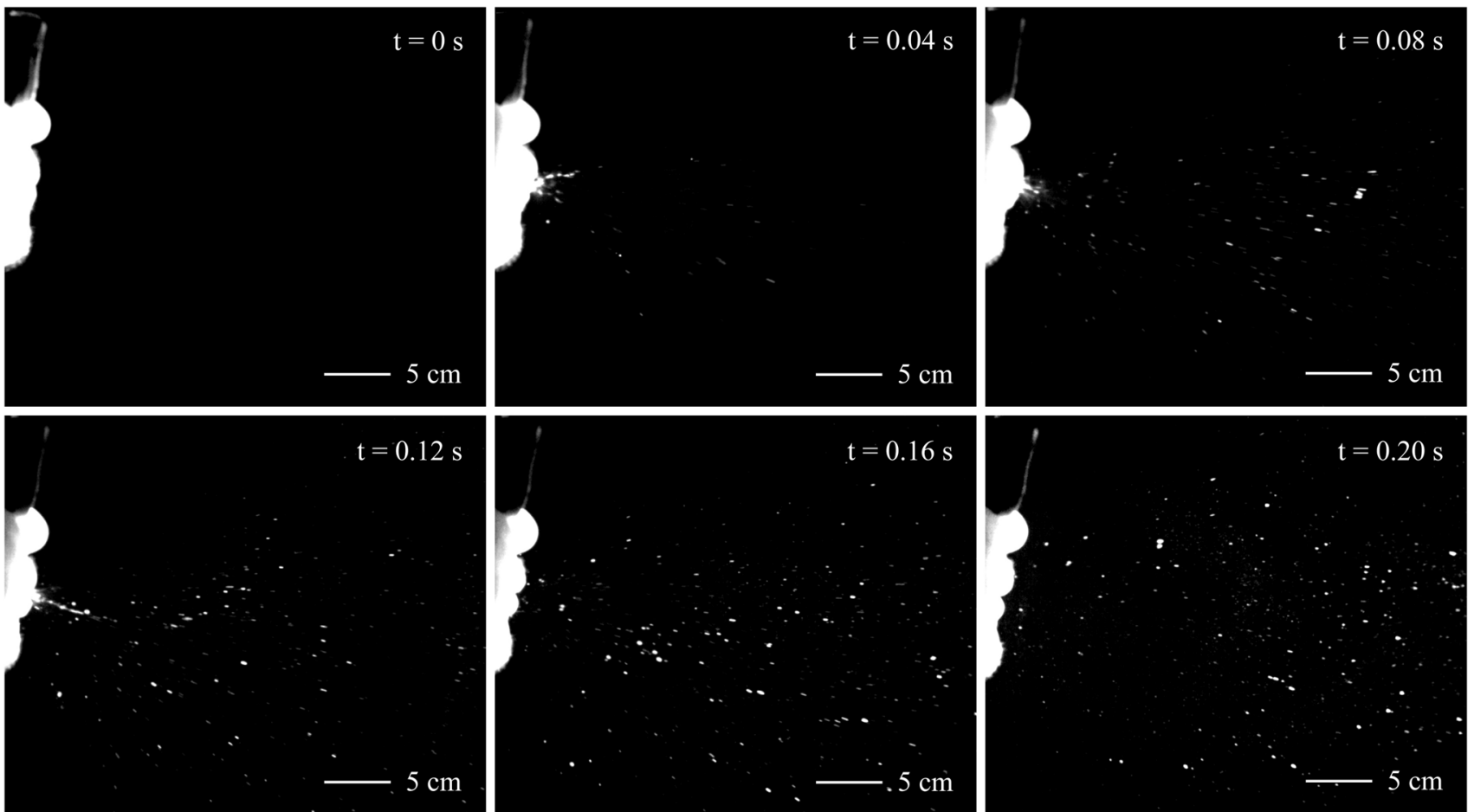

Fig. 7 Development of Sneeze. The image sequence shows multiple snapshots of sneeze 2 separated by an interval of $40 \mathrm{~ms}$

of the sneeze flow it still highlights the variability among different sneezes. Specifically, a typical sneeze lasted for approximately 180-200 $\mathrm{ms}$ and the maximum droplet

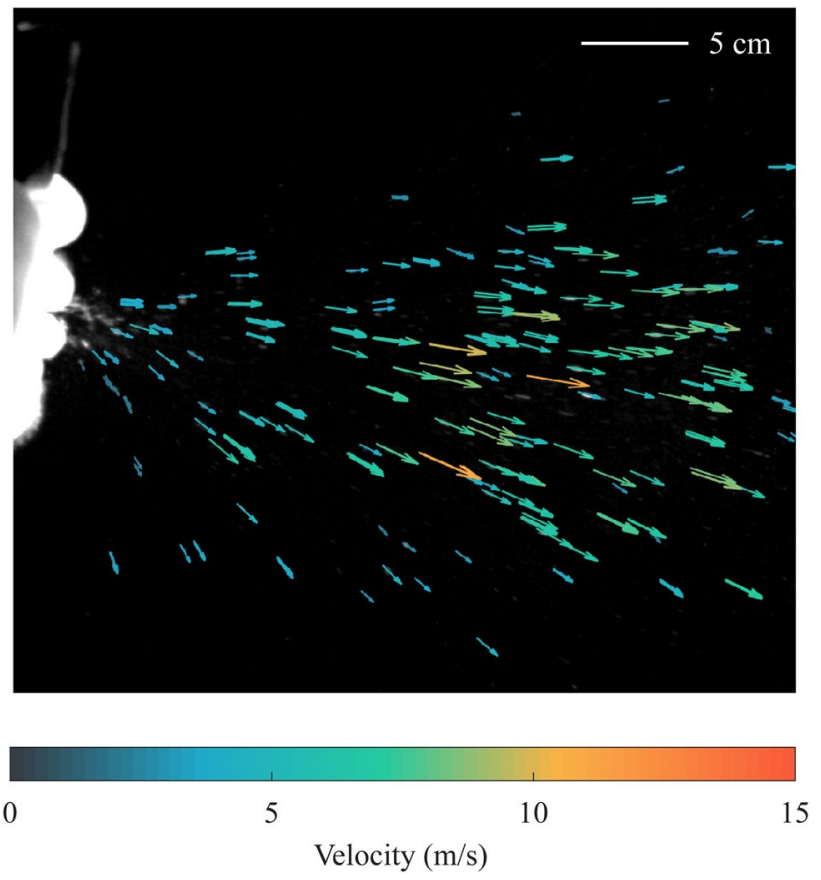

Fig. 8 Velocity vectors of expelled droplets for sneeze $2100 \mathrm{~ms}$ after the onset of sneeze velocity observed ranged from $12-15 \mathrm{~m} / \mathrm{s}$ but, the number of droplets expelled varied a lot as can be observed in Fig. 4. This can significantly influence the extent of spread of expelled droplets which is the basis of droplet infection control guidelines (World Health Organization 2020). It should be noted that the variation in expelled droplets could be partially due to the fact that only a slice of flow was illuminated, or the mouth of some subjects could be drier and produced fewer droplets. Nevertheless, it is also possible that the number of droplets expelled varies substantially between different sneezes, and hence, future works with larger sample size are required to explore this further. Moreover, the actual droplet count is also likely to depend on the level of illumination available which dictates the smallest droplets that can be detected.

Table 1 shows the mean velocity, standard deviation, and sneeze duration among the three different sneezes. In addition to this, the 99th percentile droplet velocity for Sneeze

Table 1 Mean velocity, standard deviation, and sneeze duration among 3 different sneezes shown in Fig. 4

\begin{tabular}{llll}
\hline Sneeze no. & $\begin{array}{l}\text { Mean velocity } \\
(\mathrm{m} / \mathrm{s})\end{array}$ & $\begin{array}{l}\text { Std. deviation } \\
(\mathrm{m} / \mathrm{s})\end{array}$ & Duration (s) \\
\hline 01 & 3.8 & 1.9 & 0.196 \\
02 & 3.3 & 2.0 & 0.190 \\
03 & 3.0 & 1.8 & 0.184 \\
\hline
\end{tabular}



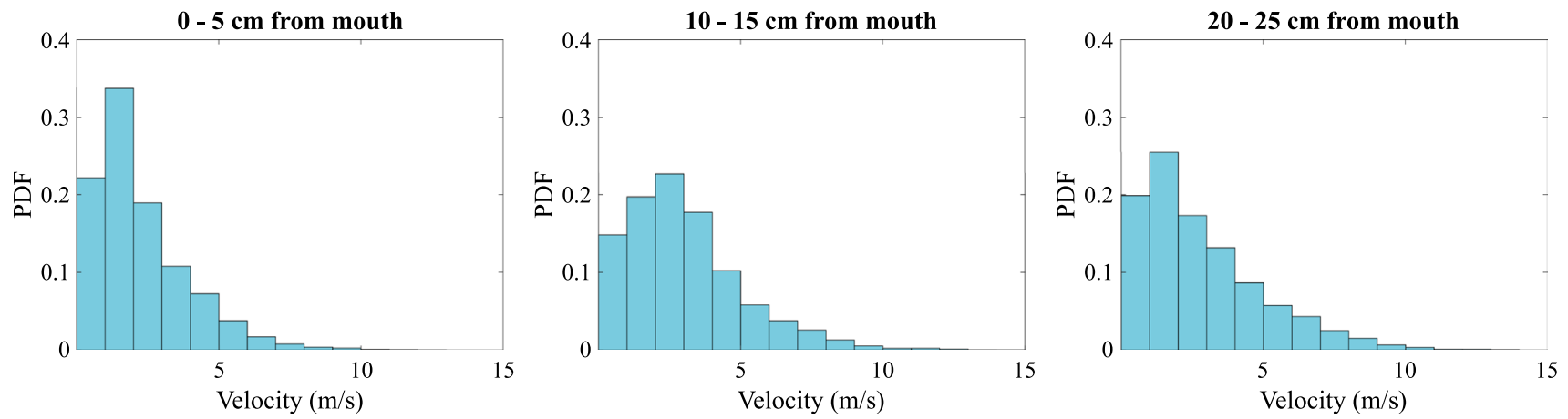

Fig. 9 Probability distribution of particle velocity vectors of sneeze 2 at three different locations from the mouth exit

1, Sneeze 2, and Sneeze 3 are $9.69 \mathrm{~m} / \mathrm{s}, 9.26 \mathrm{~m} / \mathrm{s}$, and $8.66 \mathrm{~m} / \mathrm{s}$, respectively. And 80th percentile droplet velocity for Sneeze 1, Sneeze 2, and Sneeze 3 are $5.36 \mathrm{~m} / \mathrm{s}, 4.78 \mathrm{~m} / \mathrm{s}$, and $4.77 \mathrm{~m} / \mathrm{s}$, respectively. This implies that less than $1 \%$ of the droplets expelled travel at velocities greater than $10 \mathrm{~m} / \mathrm{s}$ and almost $80 \%$ of the droplets travel at velocities slower than $5 \mathrm{~m} / \mathrm{s}$. The maximum velocities of droplets observed are in the range of $12-15 \mathrm{~m} / \mathrm{s}$, which is higher than the values reported by Nishimura et al. (2013) and Tang et al. (2013). These values are similar to the maximum droplet velocity reported by Scharfman et al. (2016) but, significantly less than the ligament velocity reported in that study. These values are also lower than those assumed by Xie et al. (2007) for modelling. The maximum estimation error in particle tracking algorithm is \pm 0.5 pixels hence, the error in the velocity estimates would be less than $\pm 0.1 \mathrm{~m} / \mathrm{s}$. To statistically determine the best conditions for modelling velocity of droplets expelled during a sneeze, further work over a significantly larger sample size would be necessary and will be the subject of a future study.

Figure 8 shows the velocity vectors of droplets expelled in sneeze 2. To quantify the expelled droplet flow-field, Fig. 9 shows the probability distribution of droplet velocity vectors at three different distances from the mouth. These locations were chosen to span our field of view and to get a quantitative measure of the velocity distribution as a function of stream-wise distance. Our field of view for the sneeze was restricted to $30 \mathrm{~cm}$ due to the head movement of the subject so, 3 points were chosen-one close to mouth, one in the middle of the field of view, and one close to the end. The results reveal an initial increase in velocity vectors of magnitude greater than $5 \mathrm{~m} / \mathrm{s}$ at a distance of $10-15 \mathrm{~cm}$ from mouth exit. Since small droplets expelled generally move with the airflow (Hinds 1999), this increase in high velocity vectors implies that either there is an increase in number of large droplets or the droplets are accelerating initially. The increase in number of droplets could be explained by the fact that a series of ligament breakup happens at the mouth exit as shown by Scharfman et al. (2016).

\section{Summary and outlook}

This study presents an experimental framework to visualize individual distinct droplets expelled during a sneeze which utilises particle tracking velocimetry to understand the droplet motion more precisely. The visualization technique used allows to locate particles without overlap along the camera axis and leads to precise droplet velocity measurements. Our findings highlight the variation in the velocity distribution of droplets expelled in different sneezes and points toward the possibility of a large variation in the number of droplets expelled, both among the sneezes by same individual and between different individuals. We observed the formation of droplets by breakup of ligaments at the mouth exit and expulsion of small particles directly from the respiratory tract. These findings highlight the shortcoming of studies trying to characterize sneeze flow and size distribution of expelled droplets with small sample size. There is also a possibility of differences in the viscoelasticity of expiratory fluid of different individuals as we observed rapid ligament to droplet conversion in sneeze 2 . This is an important factor in determining the size distribution of large droplets and the extent of their spread hence, future studies on this aspect with large datasets are also important to better understand the size distribution of the droplets generated. This study only used healthy subjects, and the composition of expiratory fluid may be different in individuals with respiratory infections. It will be valuable to study the difference in viscoelasticity and subsequently the droplet count between healthy and infected subjects, and how infection may affect a sneeze.

A limitation of visualising a planar slice of the sneeze flow is the exclusion of a significant number of droplets from the captured image sequence. As we are visualising a planar slice to accurately determine the flow speeds associated with expelled droplets, the droplets out of the plane are excluded from the analysis.

In summary, this study presents a methodology to accurately quantify the velocity field associated with droplet 
motion in sneezes. The technique is thereafter validated through applying it to multiple sneezes captured to highlight its potential, and in doing so we also observed the marked differences between the sneezes captured. These variations observed in sneeze characteristics highlights that it is essential to have a large sample size to fully characterise the droplet trajectories towards estimating and modelling the extent of spread for a general sneeze, which will be addressed in a future work that will employ a substantially larger sample size.

Acknowledgements This research was supported by NHMRC Centre for Research Excellence [Grant number: 1107393] (Integrated Systems for Epidemic Response [ISER])

\section{Compliance with ethical standards}

Conflict of interest The authors declare that they have no conflict of interest.

\section{References}

Bahl P, Doolan C, de Silva C, Chughtai AA, Bourouiba L, MacIntyre CR (2020) Airborne or droplet precautions for health workers treating coronavirus disease 2019? J Infect Dis. https://doi. org/10.1093/infdis/jiaa189

Bourouiba L (2020) Turbulent gas clouds and respiratory pathogen emissions. JAMA. https://doi.org/10.1001/jama.2020.4756

Bourouiba L, Dehandschoewercker E, Bush JW (2014) Violent expiratory events: on coughing and sneezing. J Fluid Mech 745:537563. https://doi.org/10.1017/jfm.2014.88

Cowen EA, Monismith SG (1997) A hybrid digital particle tracking velocimetry technique. Exp Fluids 22(3):199-211. https://doi. org/10.1007/s003480050038

De Silva CM, Baidya R, Khashehchi M, Marusic I (2012) Assessment of tomographic PIV in wall-bounded turbulence using direct numerical simulation data. Exp Fluids 52(2):425-440. https://doi. org/10.1007/s00348-011-1227-7

Dick EC, Jennings LC, Mink KA, Wartgow CD, Inborn SL (1987) Aerosol transmission of rhinovirus colds. J Infect Dis 156(3):442448. https://doi.org/10.1093/infdis/156.3.442

Duguid JP (1922) The and droplet-nuclei.size and duration of air-carriage of respiratory droplets. J Hyg 44:471-479

Gupta JK, Lin CH, Chen Q (2009) Flow dynamics and characterization of a cough. Indoor Air 19(6):517-525. https://doi.org/10.11 11/j.1600-0668.2009.00619.x

Hinds WC (1999) Aerosol technology: properties, behavior, and measurement of airborne particles. John Wiley \& Sons, Hoboken

Jennison MW (1942) Atomizing of mouth and nose secretions into the air as revealed by high-speed photography. In: Aerobiology, 17th edn, American Assn. for the Advancement of Science, pp $106-128$

Lee J, Yoo D, Ryu S, Ham S, Lee K, Yeo M, Min K, Yoon C (2019) Quantity, size distribution, and characteristics of cough-generated aerosol produced by patients with an upper respiratory tract infection. Aerosol Air Qual Res 19(4):840-853. https://doi. org/10.4209/aaqr.2018.01.0031
Lewis JP (1995) Fast normalized cross-correlation

Loudon RG, Brown LC (1967) Cough frequency in patients with respiratory disease. Am Rev Respir Dis 96(6):1137-1143. https:// doi.org/10.1164/arrd.1967.96.6.1137

MacIntyre CR, Chughtai AA, Seale H, Richards GA, Davidson PM (2014) Respiratory protection for healthcare workers treating Ebola virus disease (EVD): Are facemasks sufficient to meet occupational health and safety obligations? Int J Nurs Stud 51(11):1421-1426. https://doi.org/10.1016/j.jpurstu.2014.09.002

MATLAB (2019) Fuzzy logic toolbox (R2019a). The MathWorks Inc., Natick

Nishimura H, Sakata S, Kaga A (2013) A new methodology for studying dynamics of aerosol particles in sneeze and cough using a digital high-vision, high-speed video system and vector analyses. PLoS One. https://doi.org/10.1371/journal.pone.0080244

Parienta D, Morawska L, Johnson GR, Ristovski ZD, Hargreaves M, Mengersen K, Corbett S, Chao CYH, Li Y, Katoshevski D (2011) Theoretical analysis of the motion and evaporation of exhaled respiratory droplets of mixed composition. J Aerosol Sci 42(1):1-10. https://doi.org/10.1016/j.jaerosci.2010.10.005

Scharfman BE (2016) Three dimensional imaging of multiphase flows : from bubbles to sneezes. $\mathrm{PhD}$ thesis, Massachusetts Institute of Technology, http://hdl.handle.net/1721.1/103479

Scharfman BE, Techet AH, Bush JWM, Bourouiba L (2016) Visualization of sneeze ejecta: steps of fluid fragmentation leading to respiratory droplets. Exp Fluids 57(2):24. https://doi.org/10.1007/ s00348-015-2078-4

Siegel JD, Rhinehart E, Jackson M, Chiarello L (2007) Guideline for isolation precautions: preventing transmission of infectious agents in health care settings. Am J Infect Control 35(10):S65-S164. https://doi.org/10.1016/j.ajic.2007.10.007

Tang JW, Nicolle AD, Klettner CA, Pantelic J, Wang L, Suhaimi AB, Tan AYL, Ong GWX, Su R, Sekhar C, Cheong DDW, Tham KW (2013) Airflow dynamics of human jets: sneezing and breathingpotential sources of infectious aerosols. PLoS One 8(4):1-7. https ://doi.org/10.1371/journal.pone.0059970

Tropea C, Yarin AL, Foss JF (2007) Springer Handbook of Experimental Fluid Mechanics. Springer, Berlin, https://doi.org/10.1007/9783-540-30299-5

Vansciver M, Miller S, Hertzberg J (2011) Particle image velocimetry of human cough. Aerosol Sci Technol 45(3):415-422. https://doi. org/10.1080/02786826.2010.542785

World Health Organization (2014) Infection prevention and control of epidemic- and pandemic-prone acute respiratory infections in health care. http://apps.who.int/iris/bitstream/10665/11265 6/1/9789241507134_eng.pdf

World Health Organization (2020) Management of ill travellers at points of entry - international airports, seaports and ground crossings - in the context of COVID-19 outbreak: interim guidance, 16 February 2020. https://apps.who.int/iris/handle/10665/331003

Xie X, Li Y, Chwang AT, Ho PL, Seto WH (2007) How far droplets can move in indoor environments-revisiting the Wells evaporationfalling curve. Indoor Air 17(3):211-225. https://doi.org/10.111 1/j.1600-0668.2007.00469.x

Zhu SW, Kato S, Yang JH (2006) Study on transport characteristics of saliva droplets produced by coughing in a calm indoor environment. Build Environ 41(12):1691-1702. https://doi.org/10.1016/j. buildenv.2005.06.024

Publisher's Note Springer Nature remains neutral with regard to jurisdictional claims in published maps and institutional affiliations. 